\title{
Measuring Students Affective States through Online Learning Logs - An Application of Learning Analytics
}

\author{
Yang Wang, Qingtang Liu, David Stein, and Qihui Xia
}

\begin{abstract}
The affective state is determinate to online learning quality. It is related to students' attitude, learning motivation, and learning engagement. Learning affective states consists of engagement, frustration, confusion, and off-task state in this study. Different affective states are associated with different online learning behavior features. Affective states analysis consists of data collecting, data processing, affective states analyzing, evaluating, and intervening. Students' affective states can be analyzed by the affective state detectors. 12,912 online learning operations from an online course are used to construct the affective state detectors and examine the effectiveness. The consistency of the analysis results with the self-reports supported that the model is reliable to analyze students' affective state.
\end{abstract}

Index Terms-Research and application, online affective state; analysis model.

\section{INTRODUCTION}

With the rapid development of information technology, online learning has become popular as a new learning environment. It opens a new door for learners who are busy with their work and family or those with physical challenges that prevent them from learning at schools far from them. However, there are several challenges faced by online learning. For example, there are lots of online learners but few of them insisted on learning and obtained a certification [1]. There are abundant learning resources, but few satisfy learners' needs. There are numerous online learning platforms but few of them engaged learners. The lack of in-depth analysis of students' affective states is an essential factor that influences their online learning [2]. The state of students' engagement, the form of their participation, and their learning attitude are becoming the starting and driving force of online learning. In-depth analysis of the affective states has become an important topic for the popularity of online learning.

Affective states refer to a learner's physical and mental concentration on learning. Studies have shown that different affective states can produce different learning effects [3]. An intelligent tutoring system is aimed at improving students' learning based on their affective states which is a combination of cognition and emotion. Therefore, affective

Manuscript received November 11, 2018; revised March 1, 2019.

Yang Wang and Qingtang Liu are with the Central China Normal University, China (e-mail: wangyang0519@mails.ccnu.edu.cn, liuqtang@mail.ccnu.edu.cn).

David Stein is with the Ohio State University, USA (e-mail: stein.1@osu.edu).

Qihui Xia is with the Nanjing Normal University, China (e-mail: qihuixia1993@163.com). states are the starting and destination of online learning. Being actively engaged in the learning process is necessary for deep learning. The concentration state allows learners to have higher and lasting emotional, cognitive, and behavioral engagement in learning content [4]. How to use students' learning log data to analyze their affective states and support their online learning is essential to improve the online learning quality. To this end, Gartner announced a project "Reducing Gap: Transforming Data into Actions" to analyze students' affective states through educational data to achieve effective teaching [5]. Furthermore, the eCART system can provide real-time affective states assessment reports through monitoring and connecting learners' past and current learning performance. It is useful to monitor learners' progress and generate learners' reports to achieve effective teaching feedback [6]. The Chinese Ministry of Education announced the 13th Five-Year Plan for Education Informatization which states that it is necessary to actively use online educational data to enhance the quality of online education [7]. It indicated that the data-driven affective states analysis can enable instructors to know students' affective states and find out the learning problems to intervene in time. It is a guide for the formative assessment.

Studies on affective states focused on the affective states analysis, the influencing factors of the affective state, and the corresponding strategies. Moreover, the affective state analysis mainly uses the self-assessment method [8]. As for the influencing factors of affective states, the questionnaires and investigation are used. Then, the correlation analysis is performed to determine the influencing factors of affective states. With the development of learning analytics, the affective state analysis attracted researchers' attention. These researchers focused on learners' behaviors, physiological indicators, and other objective data to perform the affective state analysis [9]. As for the learners' affective states analysis based on students' learning log data need further study. This study constructed a data-driven affective states analysis model and examined its effectiveness based on 12,912 online learning data of an online course. Corresponding teaching strategies for those learners in different states were proposed through the interview.

\section{RELATED WORK}

\section{A. The Affective State}

The affective state describes a learner's mind when he or she is learning. The affective state is caused by human emotions. For example, the learning affective state is relatively relaxed when a learner is in a good mood, and his 
learning affective state is negative when the mood is irritated. The cognitive state influences the learning affective state by promoting the learner's understanding of the learning content [10]. In addition, an engagement state can also enable students to produce positive emotional states. The affective state is influenced by personal and environmental factors. The personal factor mainly refers to the individual's intrinsic desire for understanding a specific topic. Namely, the personal factor is a learner's internal cognitive and emotional characteristics. However, the environmental factor is the structural feature related to task organization [11]. The stimulation of an active affective state requires the combination of personal and environmental factors. Many researchers take affective states as learners' engagement [12]-[14]. They proposed that the degree of learning engagement is an important factor in students' learning experience which can affect their learning efficiency [15].

At present, there are many studies on online learning affective states, but different scholars have different understandings of affective states. Schmitz, Klug and Schmidt [16] proposed that the learning affective state is the learning behavior state measured at a particular time in a specific situation. Ansari, Montoya and Netzer [17] defined the learning affective state as the learner's involvement level and proposed that the learning affective state is a stable and adjustable psychological state. The learning affective state is the level of a learner's engagement which determined his learning efficiency. Yang, Cheng and Shih [18] supported that the learning affective state is essential to improve students' learning efficiency. The current studies on the online learning affective state are mainly focused on the following two aspects: 1) analysing a learner's knowledge construction process and learning ability based on the learner's online learning logs [19]; 2) analysing a learner's engagement according to the learner's physiological data, such as brain waves, eye movements, and skin electricity [20]. However, the learning affective states analysis based on online learning data is still in an infancy stage.

Studies indicated that the learning affective state can make a difference in learning [3], [21], [22]. However, studies on this topic tend to use the investigation methods, which are subjective and can only be applied in a relatively long period with a large population. This indicated that an analysis method based on students' online learning log may be more helpful to analyze students' learning affective states.

\section{B. Indicators of Learning Affective States}

The learning affective state is the reflection of a learner's cognitive and emotional states. In the current studies of the online learning affective state, learners' online operations are often overlooked [23]. However, these behaviors are important indicators of students' learning affective state. Statistical analysis of each learner's online learning duration, resource access, and the unit quiz can provide data for the quantitative analysis of their online learning affective states. The learning behavior is a learner's external manifestation of emotion and cognition. Although the learning affective state is below the surface of the iceberg which is difficult to observe directly, learning behavior data can be detected and reported to scale the learning affective state. The indicators for the concentration, frustration, confusion and off-task states are shown in Table I which is based on the study carried out by Pardos, Baker, San Pedro, Gowda and Gowda [24].

TABLE I: DESCRIPTION AND DATA INDICATORS OF EACH LEARNING AFFECTIVE STATE

\begin{tabular}{|c|c|}
\hline Dimension & Description \\
\hline Concentration & $\begin{array}{l}\text { The concentration is a state within a period of time. } \\
\text { For example, if the learner continuously answered } \\
\text { correctly, or the learner spends a lot of time after the } \\
\text { first wrong answer and finally answers this question } \\
\text { correctly. }\end{array}$ \\
\hline Frustration & $\begin{array}{l}\text { Frustration is an emotional state that occurs when the } \\
\text { learner's wrong answer rate is high. A student who } \\
\text { misses many times after viewing multiple tips is } \\
\text { considered to be frustrated. }\end{array}$ \\
\hline Confusion & $\begin{array}{l}\text { Confusion is a state in which a learner answered } \\
\text { incorrectly first and still answered incorrectly after } \\
\text { spending a long time on the problem. }\end{array}$ \\
\hline Off-Task & $\begin{array}{l}\text { Off-Task is a state in which learners seldom answer } \\
\text { questions or answer incorrectly but take a relatively } \\
\text { long time. }\end{array}$ \\
\hline
\end{tabular}

\section{METHODS}

\section{A. The Online Learning Affective State Analysis Model}

The learning affective state analysis is a process of evaluating learners' cognitive and emotional engagement level during the learning process to interpret the data generated by learners and detect potential problems. It is an important aspect of learning analytics. The existing learning affective state analysis models mainly consist of the following four types: the linear learning analysis model proposed by Siemens [25]; the continuous improvement model represented by Elias [26]; the circinate learning analytics framework supported by Ifenthaler and Tracey [27]; and a multi-factor learning analysis model proposed by SOLAR [28]. Siemens's linear model of learning analysis contains the basic elements of learning analysis. Elias' continuous improvement model reflects the circularity of the learning analysis process from the perspective of technical resources. A data-driven online learning affective states analysis model (as shown in Fig. 1) based on Siemens's learning analytical process model and Elias' continuous improvement model was constructed aiming at analyzing the learning affective state.

The data-driven learning affective states analysis model mainly consists of the online learning data acquisition, the data processing and affective states analysis, and the assessing, forecasting, and intervention of learning. The data collected during the data collecting are learners' online operations, including the correct answer rate, the number of operations, the interval between two adjacent operations, questions continuously answered correctly, previous operations involved the same problem, incorrect answers on a topic, and the number of past helps request. These data are analyzed to evaluate students' learning affective states. There are many redundant data in a large amount of online learning data collection. The goal of the data processing and learning 
affective states analysis is to mine useful information for analysis and visualization from the complex and varied online learning data. The data cleaning is the first step to check data consistency and process invalid values using the data standard of Table I and the method shown in Fig. 1. The learning affective state of each learner is determined according to their online learning data based on the online learning affective states' detector.

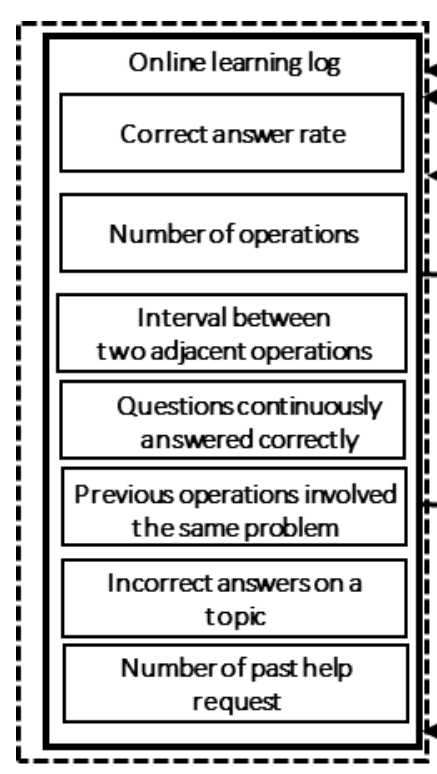

Online learning data collecting

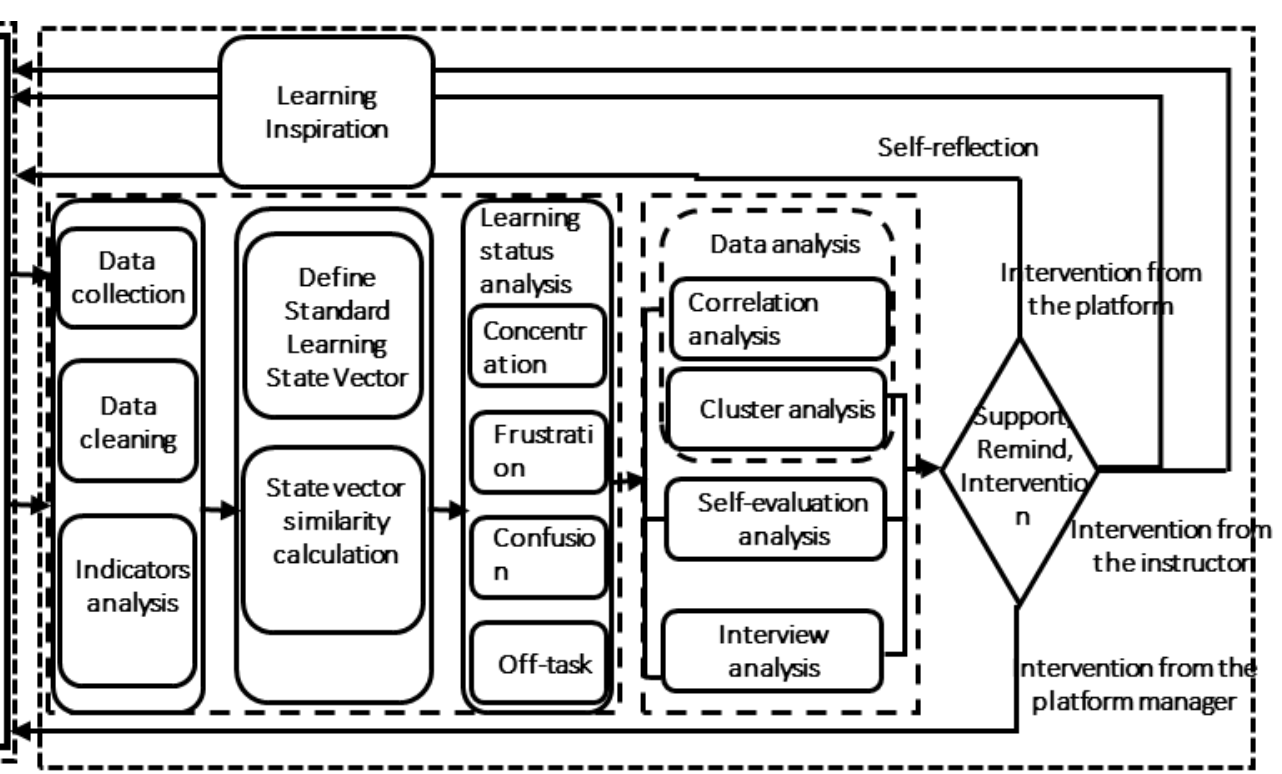

Data processing and learning status analyzing

Evaluating and Intervening

Fig. 1. Online learning affective states analysis model driven by the online learning data.

The purpose of assessing, forecasting and intervention is to engage learners in learning and maintain their active learning affective states. In this stage, the instructor is expected to intervene when learners demonstrate a negative learning affective states, such as frustration, confusion, and off-task states. For example, learners who show frustration and confusion need support in time. The presentation of corresponding knowledge and learning materials can help them to get out of frustration and confusion. For those learners who demonstrated off-task state, the appropriate monitoring, tips, and interventions are helpful. Platforms, learners, and instructors can take corresponding measures based on the results presented in the data processing and learning affective states analysis phase. For example, learners can reflect their learning behaviors, take corresponding measures, and adjust their learning affective states in time to achieve effective learning based on the presented results of the evaluation. The platform can be improved based on the analytical results. Instructors can improve teaching strategies, teaching content to optimize learning methods and stimulate learners' active learning affective states aiming at achieving adaptive teaching based on the learning affective state results. Managers can allocate resources rationally and affect the learning environment to further realize adaptive learning based on the analysis results. Through a series of interventions by learners, platforms, instructors, and managers, the online learning data, including learner-related data and learning environment-related data, will change. This can result in a cyclic process of online learning data collection, data processing, learning affective states analysis, evaluation, and intervention. It is aimed at stimulating the learner's positive learning affective states and improving their learning efficiency.

\section{A. Online Learning Affective States Analysis Method}

In the learning affective state analysis, the learning affective state detectors were used which take many indicators into account including 1) the correct answer rate; 2) number of operations; 3 ) time interval between two adjacent operations; 4) questions continuously answered correctly; 5) previous operations involving the same problem; 6) incorrect answers on a topic; 7) and the number of past help request et al. That is, the platform data was used to analyze the learning affective state. The correct answer rate is calculated according to the score of each learner in a test. The test consists of 10 questions and the full score is 100 . The number of operations is calculated according to the number of behaviors of each learner. The time interval is calculated by the learning duration divided by the number of operations. The questions continuously answered correctly is calculated by the number of questions continuously answered correctly. The learner's previous operations involved the same problem, incorrect answers on a topic, and the total number of help request is calculated according to each learner's online learning logs. The online learning platform used in this study is the Cloud Classroom developed by us which can automatically record student's learning logs including every interaction with the system.

Two hundred sixty-nine learners' 12,912 online learning operations and their learning affective states self-report results from the course "Educational information processing" from 2016 to 2018 were collected. The data was used to train and examine the effectiveness of the learning affective states analysis model. The learners are Chinese undergraduates majoring in educational technology. The "Educational information processing" is a required course for them. The 
data was shown in Fig. 2.

The self-report questionnaire for learners' online learning was constructed based on Wiebe's Learning affective states Assessment Scale [29]. There are 20 items in this scale to measure students' learning affective states. Moreover, there are 5 items in each of the following four dimensions, concentration, frustration, confusion, and off-task state [30]. Each item uses the Likert five-point scale, 1 represents strongly disagree and 5 represents strongly agree. The scale was examined to be reliable according to three online learning experts. The dimension with the highest score is the learner's main affective state. The scale was administrated to all the learners through email after the second class.

Half number $(n=135)$ of students' learning log data were used to train the model, and the other half number $(n=134)$ of students' learning data was used to examine the effectiveness of the model.

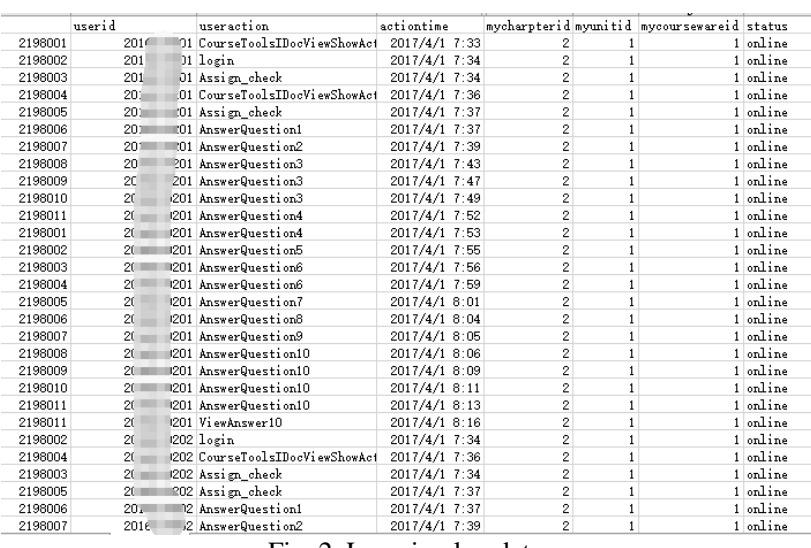

Fig. 2. Learning log data.

The detectors for the four learning affective states were developed separately (concentration- no concentration; frustration- no frustration; confusion- no confusion; and off-task-not off task). The forward selection method was used to select the appropriate features for machine learning. The features that can improve the goodness of the model will be added until no more features can be added. Finally, 24 features were included in the learning affective states detector. Cohen's Kappa was used to evaluate the detectors in which the 0 represents chance level and 1 represents perfectly. The Kappa of 0.34 means that the detector is $34 \%$ better than chance at selecting the factor. The $\mathrm{F}$ measure indicates the model accuracy in which the 0 is the worst and 1 is the best [31]. All the indicators are better than the chance as shown in Table 2. The best concentration detector was found with the Naïve Bayes which demonstrating a Kappa of .682 and an F-Measure of .570. The best frustration detector was detected using $\mathrm{K}^{*}$ algorithm with a Kappa of .368 and an F-Measure of .740. the detector of confusion was achieved with the Naive Bayes algorithm indicating a Kappa of .513 and F-Measure of .441. The detector of off-task was reached with the $\mathrm{K}^{*}$ algorithm with a Kappa of .691 and an F-Measure of .754. these indicators supported that the model is informative. The 135 students' data were used to train the learning affective states analysis model. The classifier of Naïve Bayes and $\mathrm{K}^{*}$ were used to build the model. The seven indicators of each learning affective state were collected. Results demonstrated that students in the concentration state demonstrated the following features: 1) a higher correct answer rate; 2) more operations; 3) a shorter time interval; 4) more questions continuously answered correctly; 5) more previous operations involved the same problem; 6) fewer incorrect answers on a topic; 7) and fewer past help requests. Students in frustration demonstrated the following features: 1) a lower correct answer rate; 2) more operations; 3) a shorter time interval; 4) fewer questions continuously answered correctly; 5) more previous operations involved the same problem; 6) more incorrect answers on a topic; 7) and more past help requests. Those in confusion demonstrated the following features: 1) a lower correct answer rate; 2) more operations; 3) a more time interval; 4) fewer questions continuously answered correctly; 5) more previous operations involved the same problem; 6) more incorrect answers on a topic; 7) and fewer past help requests. Students in off-task showed the following features: 1) a lower correct answer rate; 2) fewer operations; 3) more time interval; 4) fewer questions continuously answered correctly; 5) fewer previous operations involved the same problem; 6) fewer incorrect answers on a topic; 7) and fewer past help requests.

TABLE II: GOODNESS OF LEARNING AFFECTIVE STATES MODELS

\begin{tabular}{llll}
\hline \hline Affective States & Algorithm & Kappa & F-Measure \\
\hline Concentration & Naïve Bayes & 0.682 & 0.57 \\
Frustration & $\mathrm{K}^{*}$ & 0.368 & 0.74 \\
Confusion & Naïve Bayes & 0.513 & 0.441 \\
Off-Task & $\mathrm{K}^{*}$ & 0.691 & 0.752 \\
\hline \hline
\end{tabular}

The other half number $(n=134)$ of students' learning log data was used to examine the affective state detectors. These learners' operating data was analyzed using the method above. The symmetric measures results of the learning affective state self-reports and learning log analysis demonstrated that the overall results of the two methods are consistent (Kappa $=0.871, p<.001)$. Though there are 22 inconsistencies between the analysis results and the self-report results, the consistency between the affective state analysis and the self-report reached $83.6 \%$ which demonstrated that the data analysis model is valid.

\section{B. Interview}

Two students in each learning affective state (concentration, frustration, confusion, and off-task state) were selected randomly for an interview. Namely, eight students were interviewed. The interview was mainly conducted from the following four aspects, engagement, satisfaction, perceived difficulty and expected support. Students in concentration showed a high level of engagement, satisfaction, and a low perceived difficulty. They thought that resources in the learning platform were comprehensive. Although the basic functions of the system can satisfy students' needs, they proposed that the resources can be refined which would help them find useful learning materials quickly. Students in the frustration and confusion states demonstrated a moderate level of engagement and learning satisfaction, a higher level of perceived difficulty compared with those in the concentration state. They suggested that the tips could be more detailed. In terms of expected support, 
they expressed that the analysis of topics could be more detailed and instructor's timely help was beneficial for them. Besides, they expected that the real-time learning affective states visualization would help them realize the problem and adjust in time. Students in the off-task state showed a low level of learning engagement and satisfaction. Their perceived difficulty is moderate. They argued that it is easily distracted when faced with computers.

\section{DISCUSSION AND CONCLUSION}

The analysis results can inform online instructors about students learning affective states. It can also provide new ideas for online learning affective states analysis. That is, making full used of students' online learning log data to analyze their learning affective states and give them corresponding feedback. It is very valuable to explore the influencing factors and data indicators of different online learning affective states. Compared with existing studies, this study analyzed students learning affective states through their learning log data. The leaning state analysis model was examined to be reliable.

There are some implications in this study for the teaching strategies for different learning affective states students. For example, one student whose learning affective state is concentration proposed that the resources of the learning management system are difficult to find. It indicates that these resources could be refined and categorized clearly. One male student whose learning affective state is frustration said that he is thirsty for the teachers' feedback when he cannot understand the tips. Another male student in confusion expressed a similar opinion. He thought if the confusion cannot be solved in time, he will lose his interest to move on. As for the male student in the off-task state, he wants to play the game when he faced with the computer. "There is no teacher here, I thought I can play first", he said. The stduents in the four learning affective states can represent many online learners situation. Their feedback can give us more suggestions on how to improve our online teaching.

In addition to the correct answer rate, the number of operations, and the items mentioned above, there are many other valuable learning logs such as the number of postings and reply content. These indicators are also valuable for further learning analytics. In the future study, more online learning operations can be collected to examine the effectiveness of the learning analysis model. The relationship of the learning affective states with their learning effectiveness can be further explored to inform instructors about the necessity of appropriate intervene for students' learning. It is a guide for adaptive online teaching and an important force in facilitating personalized teaching.

\section{ACKNOWLEDGMENT}

This study was funded by Ministry of education - Chinese mobile research fund project, "Research and experiment on Regional Teaching and research supported by information technology"(No: MCM20170502) and Hubei Province Technology Innovation special projects "Key technologies and demonstration applications of Internet + Precision
Education"(No.2017ACA105).

\section{REFERENCE}

[1] K. Ng, "Implementation of new communication tools to an online chemistry course," Journal of Educators Online, vol. 15, no. 1, p. n1, 2018.

[2] M.-S. Jian and J.-L. Chen, "Individual learner big data based cloud role player game learning," Intelligent Data Analysis, vol. 21, no. S1, pp. S41-S53, 2017.

[3] M. M. T. Rodrigo, R. S. Baker, M. C. Jadud, A. C. M. Amarra, T. Dy, M. B. V. Espejo-Lahoz, S. A. L. Lim, S. A. Pascua, J. O. Sugay, and E. S. Tabanao, "Affective and behavioral predictors of novice programmer achievement," pp. 156-160.

[4] Y. Li and R. M. Lerner, "Interrelations of behavioral, emotional, and cognitive school engagement in high school students," Journal of Youth and Adolescence, vol. 42, no. 1, pp. 20-32, 2013.

[5] J. R. Evans, and C. H. Lindner, "Business analytics: The next frontier for decision sciences," Decision Line, vol. 43, no. 2, pp. 4-6, 2012.

[6] E. M. Queiroga, C. Cechinel, R. M. Araújo, and G. C. Bretanha, "Generating models to predict at-risk students in technical e-learning courses," pp. 1-8.

[7] W. Pang and J. A. Plucker, "Recent transformations in China's economic, social, and education policies for promoting innovation and creativity," The Journal of Creative Behavior, vol. 46, no. 4, pp. 247-273, 2012.

[8] T. M. Miller and L. Geraci, "Training metacognition in the classroom: The influence of incentives and feedback on exam predictions," Metacognition and Learning, vol. 6, no. 3, pp. 303-314, 2011.

[9] J. Qiu, J. Tang, T. X. Liu, J. Gong, C. Zhang, Q. Zhang, and Y. Xue, "Modeling and predicting learning behavior in MOOCs," pp. 93-102.

[10] W. Kintsch, "Learning from text, levels of comprehension, or: Why anyone would read a story anyway," Poetics, vol. 9, no. 1-3, pp. 87-98, 1980 .

[11] Z. Wang, C. Bergin, and D. A. Bergin, "Measuring engagement in fourth to twelfth grade classrooms: The classroom engagement inventory," School Psychology Quarterly, vol. 29, no. 4, pp. 517, 2014

[12] T. Dragon, I. Arroyo, B. P. Woolf, W. Burleson, R. El Kaliouby, and H. Eydgahi, "Viewing student affect and learning through classroom observation and physical sensors," pp. 29-39.

[13] Y. L. Hu, G. S. Ching, and P. C. Chao, "Taiwan student engagement model: Conceptual framework and overview of psychometric properties," International Journal of Research Studies in Education, vol. 1, no. 1, pp. 69-90, 2012.

[14] M. T. Wang and J. Degol, "Staying engaged: Knowledge and research needs in student engagement," Child Development Perspectives, vol. 8 , no. 3, pp. 137-143, 2014

[15] D. J. Shernoff, Optimal Learning Environments to Promote Student Engagement, Springer, 2013.

[16] B. Schmitz, J. Klug, and M. Schmidt, "Assessing self-regulated learning using diary measures with university students," Handbook of Self-regulation of Learning and Performance, pp. 251-266, 2011.

[17] A. Ansari, R. Montoya, and O. Netzer, "Dynamic learning in behavioral games: A hidden Markov mixture of experts approach," Quantitative Marketing and Economics, vol. 10, no. 4, pp. 475-503, 2012.

[18] M. T. Yang, Y. J. Cheng, and Y. C. Shih, Facial Expression Recognition for Learning Status Analysis, pp. 131-138.

[19] Y. Charband and N. J. Navimipour, "Online knowledge sharing mechanisms: A systematic review of the state of the art literature and recommendations for future research," Information Systems Frontiers, vol. 18, no. 6, pp. 1131-1151, 2016.

[20] C.-H. Wu, B.-C. Kuo, and G.-H. Tzeng, "Investigation of time interval size effect on SVM model in emotion norm database," New Trends in Intelligent Information and Database Systems, pp. 121-128, Springer, 2015.

[21] R. S. J. D. Baker, "Modeling and understanding students' off-task behavior in intelligent tutoring systems," pp. 1059-1068.

[22] M. Cocea, A. Hershkovitz, and R. S. Baker, "The impact of off-task and gaming behaviors on learning: Immediate or aggregate"?

[23] M. A. Conde, R. Colomo-Palacios, F. J. García-Peñalvo, and X. Larrucea, "Teamwork assessment in the educational web of data: A learning analytics approach towards ISO 10018," Telematics and Informatics, vol. 35, no. 3, pp. 551-563, 2018.

[24] Z. A. Pardos, R. S. Baker, M. O. San Pedro, S. M. Gowda, and S. M. Gowda, "Affective states and state tests: Investigating how affect throughout the school year predicts end of year learning outcomes," pp. 117-124. 
[25] G. Siemens, "1st international conference on learning analytics and knowledge 2011 (LAK'11)," 2011.

[26] T. Elias, "Learning analytics," Learning, 2011.

[27] D. Ifenthaler and M. W. Tracey, "Exploring the relationship of ethics and privacy in learning analytics and design: Implications for the field of educational technology," Educational Technology Research and Development, vol. 64, no. 5, pp. 877-880, 2016.

[28] G. Siemens, D. Gasevic, C. Haythornthwaite, S. P. Dawson, S. Shum, R Ferguson, E. Duval, K. Verbert, and R. Baker, "Open Learning Analytics: An integrated \& modularized platform," 2011.

[29] E. N. Wiebe, A. Lamb, M. Hardy, and D. Sharek, "Measuring engagement in video game-based environments: Investigation of the User Engagement Scale," Computers in Human Behavior, vol. 32, pp. 123-132, 2014.

[30] Author, Details Removed for Peer Review, 2016.

[31] C. J. Van Rijsbergen, "Foundation of evaluation," Journal of Documentation, vol. 30, no. 4, pp. 365-373, 1974.

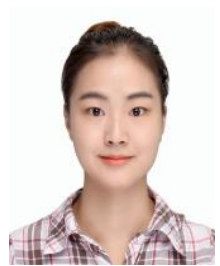

Yang Wang was born in China in May 1991. Yang earned her bachelor degree in educational technology in Hubei Normal University in 2014. Yang is a Ph.D. candidate at the School of Educational Information Technology at Central China Normal University (CCNU) and now a visiting scholar at the Ohio State University (OSU). Her research interests include learning analytics, technology-supported teaching and learning. She has published more than 7 papers in this field including in the British Journal of Educational Technology, 2018, Distance Education, 2017, and IEEE Consumer Electronics Magazine 2018.

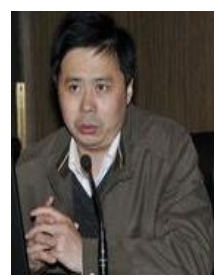

Qingtang Liu was born in China in February 1969. He is a professor at the School of Educational Information Technology at CCNU. He earned the doctoral degree in Huazhong University of Science and Technology in 2005. His recent research interests include technology-enhanced teaching and learning. He has published more than 60 papers in these fields including the Computers and Education, 2017; Journal of Educational Computing Research, 2015; the British Journal of Educational Technology, 2018 .
Dr. Liu is currently a member of ISO/IEC, a member of AVS Standards Organization, a member of Chinese E-Learning Technology Standardization Committee (CELTSC); a member of Chinese Higher Education Technology Association, a member of Chinese Education Management Information Committee.

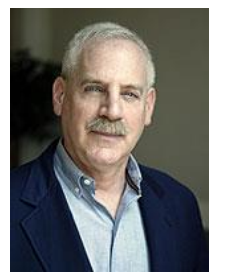

David Stein is an associate professor of College of Education and Human Ecology at the Ohio State University. He earned his doctor degree in the University of Michigan in 1976. His work centers on online teaching and learning. His recent research has focused on how learners make decisions concerning participation, choice, and use of technology in learning online settings. He has published more than 131 research papers and books including a book in 2015, the Internet and Higher Education (2013), American Journal of Distance Education (2011), and Distance Learning (2013).

Dr. Stein is currently an associate professor in the Ohio State University (OSU). He is a chair of the American Association for Adult and Continuing Education from 2014 to 2016. He earned an Imogene Oakes Award for Outstanding Research in Adult Education in 2013. He was a co-chair of the Commission on Distance Learning and Technology of the American Association for Adult and Continuing Education from 2014 to 2016.

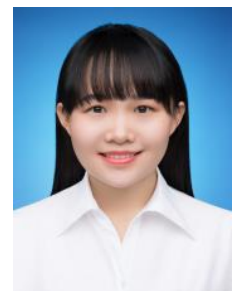

Qihui Xia was born in China in January 1993. Qihui gets her master degree of psychology at Nanjing Normal University in 2018. Her research interests include the development and educational psychology, children's theory of mind development. Her paper "Just World Belief: Conception, measurement and related research" obtained the second prize in the 17 th National Forum on the Applied Research of Psychological Techniques and the 2016 Academic Conference of Jiangsu Psychological Society in November 2016. She obtained the third prize in 2013 Jiangxi Province College Student Technology Innovation and Vocational Skills Competition in December 2013. 